\title{
Active Noise Control with a Moving Evaluation Point*
}

\author{
Ikuma IKEDA**, Shinya KIJIMOTO**, \\ Koichi MATSUDA** and Yosuke KOBA** \\ ** Kyushu University \\ 744 Motooka, Nishi-ku, Fukuoka-shi, Fukuoka 819-0395, Japan \\ E-mail: ikuma@sky.mech.kyushu-u.ac.jp
}

\begin{abstract}
Active noise control (ANC) in 3-dimensional sound field, e.g. an office room, is investigated in this paper. Since the size of the controlled area normally depends on the wave length of the target noise, it is difficult to control in the whole room using ANC technique. Instead, around-head-control is investigated in this paper. To realize the around-head-control, an evaluation point is required to correspond with the head movement while controlling. However, the evaluation point movement while controlling has not been considered in a conventional ANC. Against this problem, a new algorithm is proposed in this paper. The algorithm updates filters by using Time-Delay filter and Distance-Attenuation coefficient. Time-Delay filter and Distance-Attenuation coefficient are calculated from the position of the evaluation point. The computer simulations are carried out, and the validity of the new algorithm is shown.
\end{abstract}

Key words : Active Noise Control, LMS Algorithm, Direct Adaptive Algorithm, Moving Evaluation Point, Time-Delay, Distance-Attenuation

\section{Introduction}

In this paper, active noise control (ANC) in 3-dimensional sound field is investigated. It is difficult to realize whole-room-control because size of controlled area depends on wave length of target noise. Instead of whole-room-control, around-head-control is investigated in this paper. In this system, an evaluation point (an error microphone) and the controlled area need to follow the head movement. In the conventional ANC, Filtered- $x$ LMS algorithm ${ }^{(1)}$ is generally used because of its stability and simplicity. Since this algorithm is supposed that a secondary path characteristic (the acoustic path from the secondary sound source to an evaluation point) is constant, it is difficult to use Filtered- $x$ LMS against the evaluation point movement problem.

In 1-dimensional sound field, against this problem, Iida et al. proposed Time-delay Separation Method in 1-dimensional acoustic field ${ }^{(2)}$ and Sano et $a l^{(3)}$ proposed Direct Adaptive Algorithm (DAA). Time-delay Separation Method updates main characteristic and Time-Delay characteristic separatery. This method obtain good control effect during moving. DAA carries out both ANC and identification of the secondary path characteristic simultaneously. This feature is available in practical use. However, enough control effect is not obtained while the evaluation point is moving because LMS algorithm in DAA is insufficient in speed.

Against this problem, a new algorithm extending these method for 3-dimensional sound field is proposed in this paper. This new algorithm expresses the controller as the function of the evaluation point location. Computer simulations are carried out and results show validity of the algorithm. 


\section{Nomenclature}

\begin{tabular}{|c|c|}
\hline$a_{d}$ & Distance-Attenuation coefficient \\
\hline$c$ & Control signal from $C$ \\
\hline$C$ & Adaptive filter in DAA \\
\hline $\boldsymbol{C}_{\text {opt }}$ & Optimal filter coefficients of $\boldsymbol{C}$ \\
\hline $\boldsymbol{C}_{p}$ & The filter updated by using optimal phase characteristics \\
\hline$C_{s}$ & Enough convergent filter $\boldsymbol{C}$ at move start point \\
\hline$d$ & Desired signal (noise) \\
\hline$D$ & Adaptive filter in DAA \\
\hline $\boldsymbol{D}_{\text {opt }}$ & Optimal filter coefficients of $\boldsymbol{D}$ \\
\hline $\boldsymbol{D}_{p}$ & The filter updated by using optimal phase characteristics \\
\hline $\boldsymbol{D}_{s}$ & Enough convergent filter of $\boldsymbol{D}$ at move start point \\
\hline$e$ & Error signal \\
\hline$e_{y}$ & Control sound \\
\hline$e_{1}, e_{2}$ & Virtual error signal in DAA \\
\hline$E E$ & Estimation error \\
\hline$G$ & Secondary path characteristics \\
\hline $\boldsymbol{h}$ & Time-Delay filter \\
\hline$k$ & Sampling time \\
\hline$K$ & Adaptive filter in DAA \\
\hline $\boldsymbol{K}_{\text {opt }}$ & Optimal filter coefficients of $\boldsymbol{K}$ \\
\hline $\boldsymbol{K}_{p}$ & The filter updated by using optimal phase characteristics \\
\hline $\boldsymbol{K}_{s}$ & Enough convergent filter of $\boldsymbol{K}$ at move start point \\
\hline$l$ & Distance between the evaluation point and the position of 2 nd sound source \\
\hline$l_{s}$ & Distance between the move start point and the position of 2 nd sound source \\
\hline $\boldsymbol{r}$ & Reference signal vector \\
\hline$s$ & Output signal from $\boldsymbol{K}$ \\
\hline$V$ & Sound velocity \\
\hline $\boldsymbol{W}$ & Primary path characteristics \\
\hline $\boldsymbol{W}_{\text {ref }}$ & Acoustic characteristics from primary sound source to reference microphone \\
\hline$(x y)$ & Position of the evaluation point \\
\hline$\left(x_{s} y_{s}\right)$ & Position of the move start point \\
\hline$\gamma$ & Normalized step size parameter \\
\hline$\mu$ & Weight coefficient \\
\hline
\end{tabular}

\section{Identification of acoustic characteristics and optimal filter coefficients}

Identified secondary path characteristic $\hat{\boldsymbol{G}}$ is necessary in the conventional Filtered- $x$ LMS algorithm. If the identifying error is large, the controller does not work properly by this algorithm. On the other hand, DAA updates three filters $\boldsymbol{C}, \boldsymbol{D}$ and $\boldsymbol{K}$ simultaneously using LMS algorithm as shown in Fig. 1. Since filter $\boldsymbol{K}$ is identified as $\boldsymbol{G}$ during ANC, the prior estimation of $\boldsymbol{G}$ is unnecessary. However, even using DAA, accuracy of identification becomes worse if the evaluation point moves.

Measurements are carried out to investigate changes of acoustic characteristics while the evaluation point is moving. An experimental arrangement in this paper are shown in Fig. 2. The acoustic characteristics in experimental field are identified as FIR filters using adaptive identification. The conditions of identification are shown in Table 1. Identified $\boldsymbol{W}$ and $\boldsymbol{G}$ at the origin are shown in Fig. 3. Figure 4 shows $\boldsymbol{W}$ along $x$ - and $y$-axes and Fig. 5 shows $\boldsymbol{G}$ along $x$ - and $y$ - axes. The vertical axis in Fig. 4 and 5 expresses sampling number from 40 to 60 for $\boldsymbol{W}$, and from 10 to 35 for $\boldsymbol{G}$ that enlarges horizontal axis in Fig. 3. In Fig. 4 and 5, the color expresses the amplitude and the horizontal axis expresses $x$-coordinate or $y$ coordinate. Figures 4 and 5 show that the difference of acoustic characteristics mainly appears in the difference of Time-Delay. 
Computer simulations are carried out in order to inspect the changes of optimal characteristics of controller. Optimal filter coefficients $\boldsymbol{C}_{\mathrm{opt}}, \boldsymbol{D}_{\mathrm{opt}}$ and $\boldsymbol{K}_{\mathrm{opt}}$ at each point are calculated from the identified $\boldsymbol{W}$ and $\boldsymbol{G}$. $\boldsymbol{C}_{\mathrm{opt}}, \boldsymbol{D}_{\mathrm{opt}}$ and $\boldsymbol{K}_{\mathrm{opt}}$ along $x$ - and $y$-axes are shown in Fig. 6, 7 and 8. The difference of optimal filters mainly appears in Time-Delay the same as $\boldsymbol{W}$ and $\boldsymbol{G}$. In order to check this feature, frequency responses of $\boldsymbol{C}_{\mathrm{opt}}$ at points $(00),(0.020),(0.04$ $0)$ and $(0.060)$ are calculated. From Fig. 9, while the phase shift is observed obviously, the amplitude difference is small. From these results, it is considered that the phase adjusting, i.e. Time-Delay updating, is more effective than the amplitude adjusting while the evaluation point moves.

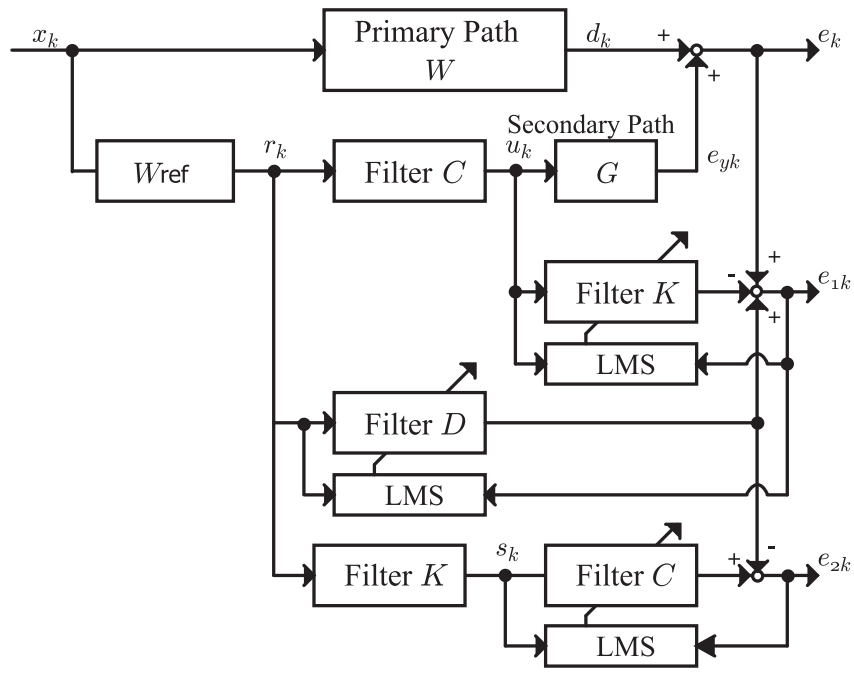

Fig. 1 Block diagram of Direct Adaptive Algorithm

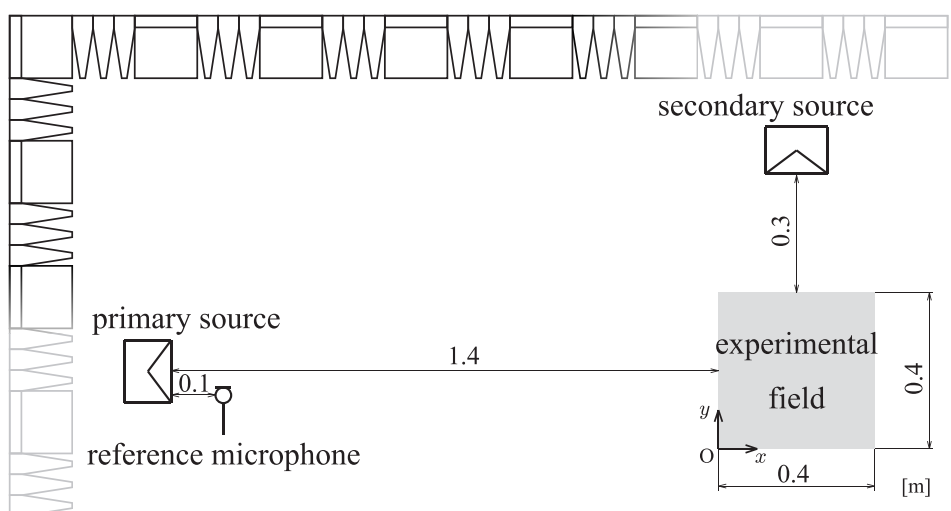

Fig. 2 Experimental arrangement

Table 1 Conditions of identifying acoustic characteristics

\begin{tabular}{|c|c|}
\hline Temperature & $297 \sim 300 \mathrm{~K}$ \\
\hline Used algorithm & Normalized LMS \\
\hline Step size & 0.01 \\
\hline Sampling frequency & $10 \mathrm{kHz}$ \\
\hline Filter length & 512 \\
\hline Identifying time & $30 \mathrm{~s}$ \\
\hline Input signal & white noise \\
\hline position of identified point & $(0.02 m, 0.02 n), m, n=0,1,2 \cdots 20$ \\
\hline number of identifying points & $441(=21 \times 21)$ \\
\hline
\end{tabular}




\section{Dynamics}
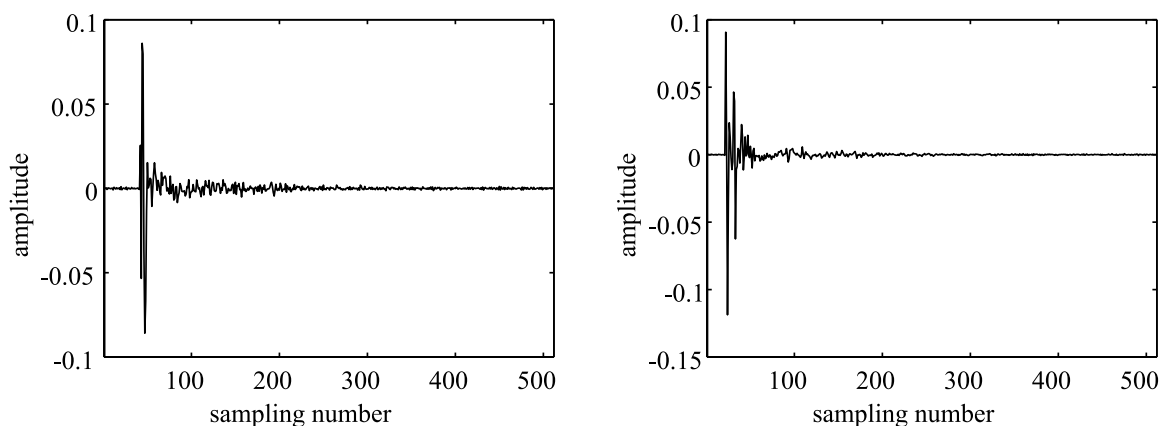

Fig. 3 Identified $\boldsymbol{W}$ (left) and $\boldsymbol{G}$ (right) at point $\left(\begin{array}{ll}0 & 0\end{array}\right)$
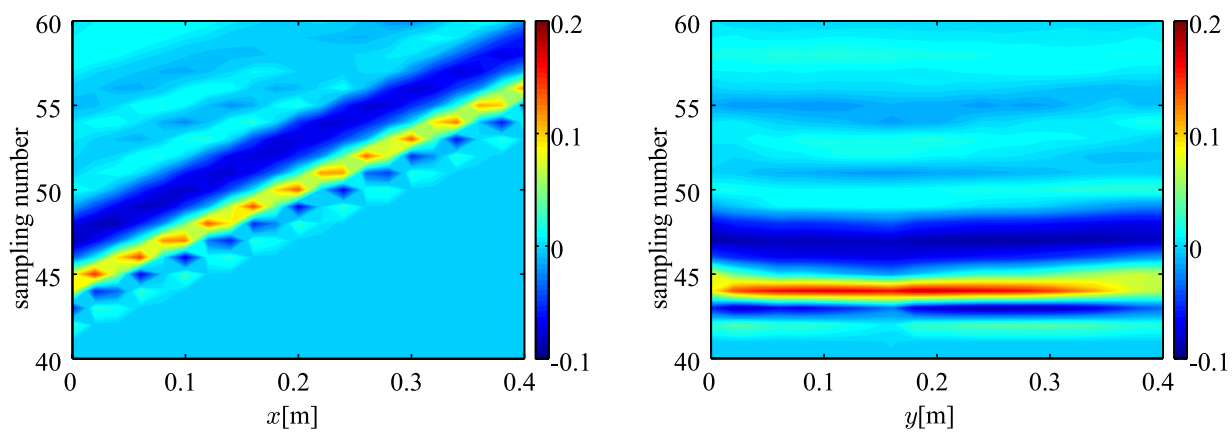

Fig. 4 Identified primary path characteristics $W$ along $x$-axis(left) Identified primary path characteristics $\boldsymbol{W}$ along $y$-axis(right)
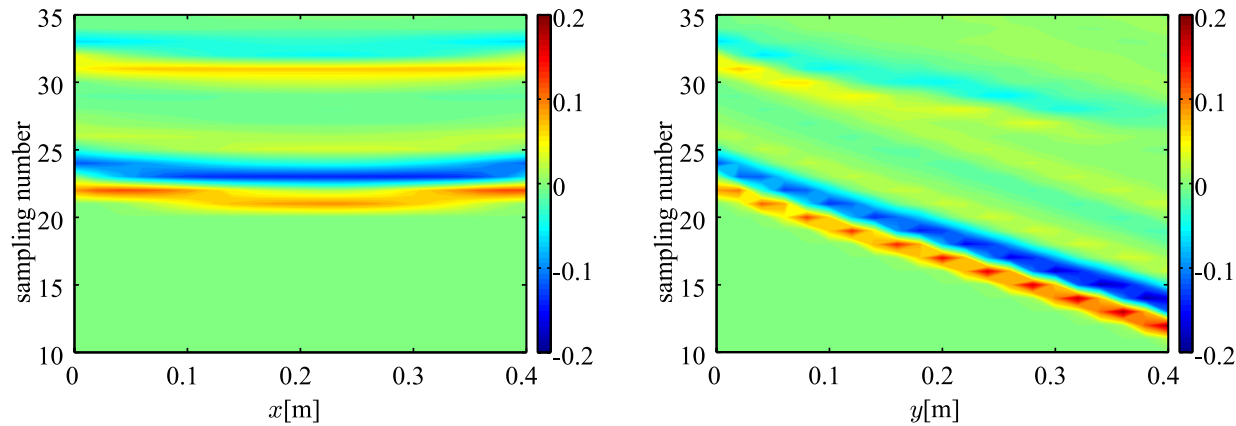

Fig. 5 Identified secondary path characteristics $\boldsymbol{G}$ along $x$-axis(left) Identified secondary path characteristics $\boldsymbol{G}$ along $y$-axis(right)
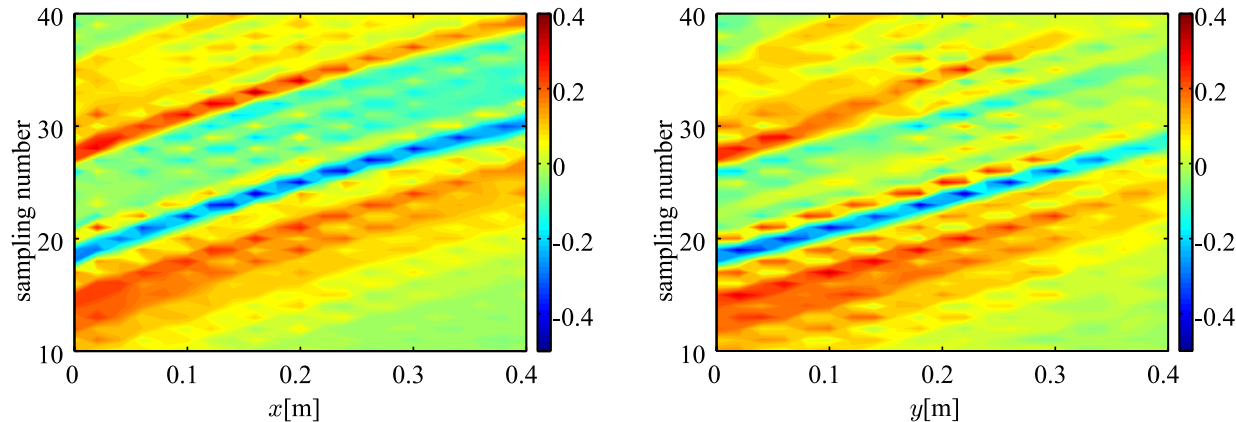

Fig. 6 Optimal filter coefficients $\boldsymbol{C}_{\text {opt }}$ along $x$-axis(left) Optimal filter coefficients $\boldsymbol{C}_{\text {opt }}$ along $y$-axis(right) 

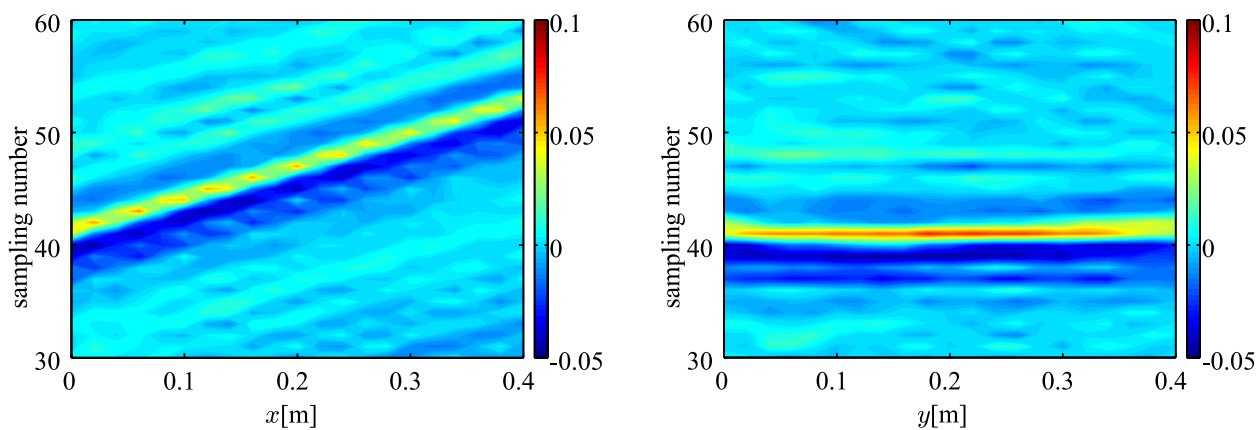

Fig. 7 Optimal filter coefficients $\boldsymbol{D}_{\text {opt }}$ along $x$-axis(left) Optimal filter coefficients $\boldsymbol{D}_{\text {opt }}$ along $y$-axis(right)
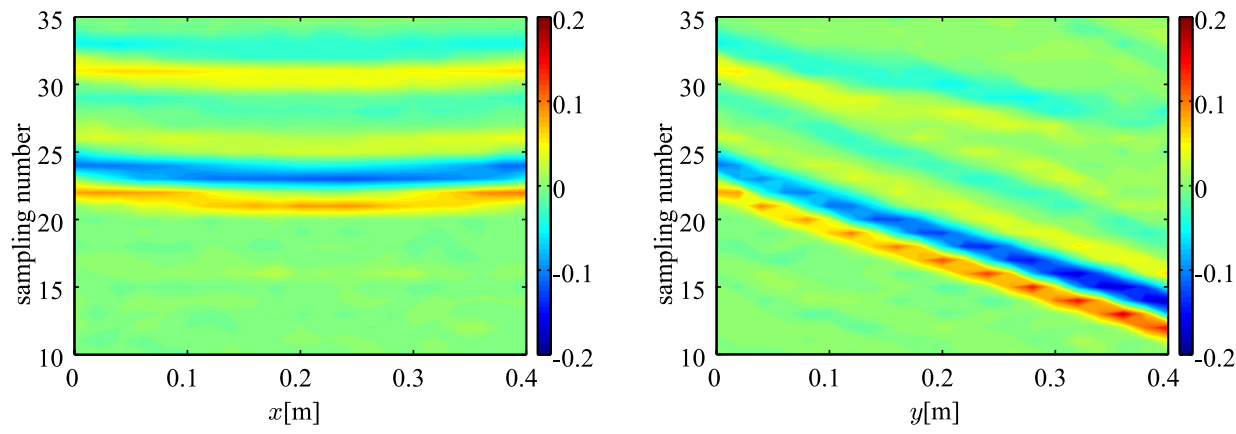

Fig. 8 Optimal filter coefficients $\boldsymbol{K}_{\text {opt }}$ along $x$-axis(left) Optimal filter coefficients $\boldsymbol{K}_{\mathrm{opt}}$ along $y$-axis(right)

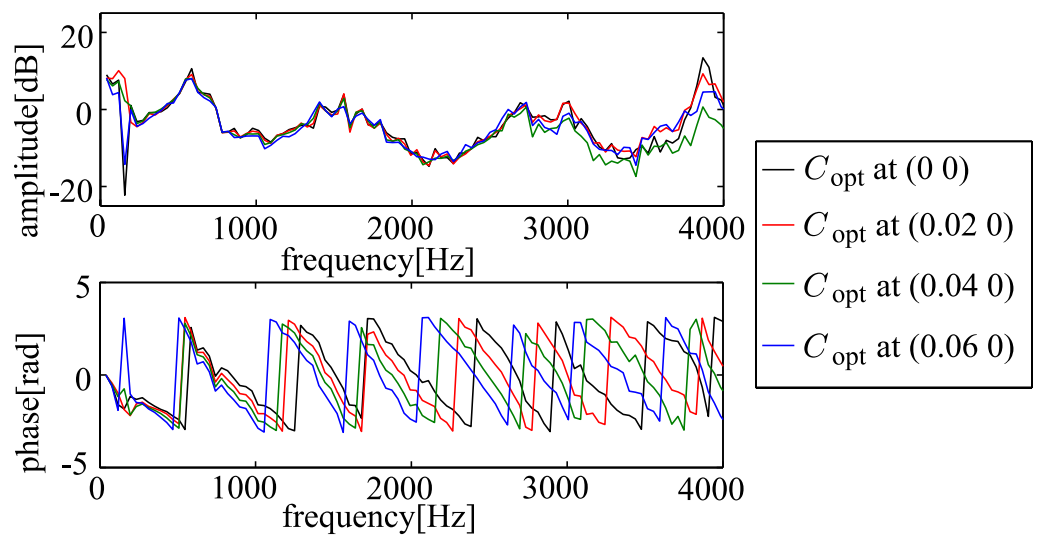

Fig. 9 Frequency response of $\boldsymbol{C}_{\text {opt }}$ at $(00) \sim(0.060)$

\section{ANC simulations}

In this section, several ANC simulations assuming movement of the evaluation point are carried out. In the simulations, acoustic characteristics obtained in $\S 3$ are used. Figure 10 shows the movement of the evaluation point. Table 2 shows the conditions of simulations. Estimation error $(E E)$ expressed in Eq. 1 is used in order to evaluate the control effect. In this paper, $q$ is set to 512 in Eq. 1.

$$
E E_{k}=10 \log _{10}\left(\frac{\sum_{n=k-q+1}^{k} e_{n}^{2}}{\sum_{n=k-q+1}^{k} d_{n}^{2}}\right) .
$$




\subsection{Interpolating the optimal filters}

Figure 11 shows a simulation result of DAA with linear-interpolated optimal filters calculated in $\S 3$. The enough control effect was obtained by this method, while the control effect became worse by DAA just after the evaluation point starts moving. This method is optimal, however, because prior identifications are necessary to use this method. A method without a prior identification should be invented.

\subsection{Geometrical estimation of Time-Delay and Distance-Attenuation}

As described in $\S 3$, the optimal filters has related to the position of the evaluation point. In the new method, the filters are expressed as functions of location as follows,

$$
\begin{aligned}
& \boldsymbol{C}_{(x y)}=a_{d C(x y)}\left(\boldsymbol{C}_{s} * \boldsymbol{h}_{C(x y)}\right)-2 \gamma_{C k} e_{2 k} \boldsymbol{s}_{k} \\
& \boldsymbol{D}_{(x y)}=a_{d D(x y)}\left(\boldsymbol{D}_{s} * \boldsymbol{h}_{D(x y)}\right)-2 \gamma_{D k} e_{1 k} \boldsymbol{r}_{k} \\
& \boldsymbol{K}_{(x y)}=a_{d K(x y)}\left(\boldsymbol{K}_{s} * \boldsymbol{h}_{K(x y)}\right)+2 \gamma_{K k} e_{1 k} \boldsymbol{u}_{k}
\end{aligned}
$$

In Eq. 2, $a_{d C(x y)}, a_{d D(x y)}$ and $a_{d K(x y)}$ are calculated from Distance-Attenuation of noise and control sound, and $\boldsymbol{h}_{C(x y)}, \boldsymbol{h}_{D(x y)}$ and $\boldsymbol{h}_{K(x y)}$ are calculated from Time-Delay of noise and

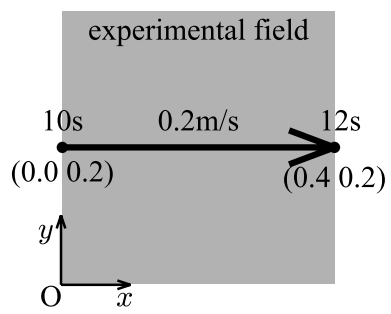

Fig. 10 The movement of the evaluation point

Table 2 Conditions of ANC simulations

\begin{tabular}{|c|c|}
\hline Temperature & $297 \mathrm{~K}$ \\
\hline Used algorithm & DAA, DAA with proposed \\
\hline Step size of DAA & 100 \\
\hline Sampling frequency & $10 \mathrm{kHz}$ \\
\hline Filter length of adaptive filters & 256 \\
\hline Input signal & white noise (up to $4 \mathrm{kHz}$ ) \\
\hline
\end{tabular}

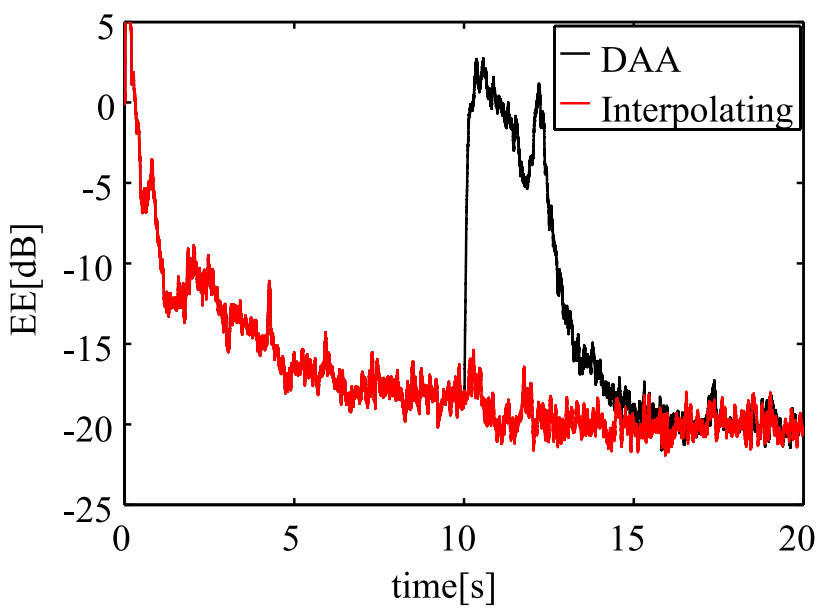

Fig. 11 A simulation controlled by interpolating the optimal filters 
control sound. $\boldsymbol{C}_{\mathrm{opt}}, \boldsymbol{D}_{\mathrm{opt}}$ and $\boldsymbol{K}_{\mathrm{opt}}$ are written

$$
\left.\begin{array}{l}
\boldsymbol{C}_{\mathrm{opt}}=-\frac{\boldsymbol{W}}{\boldsymbol{W}_{\mathrm{ref}} \boldsymbol{G}} \\
\boldsymbol{D}_{\mathrm{opt}}=-\frac{\boldsymbol{W}}{\boldsymbol{W}_{\mathrm{ref}}} \\
\boldsymbol{K}_{\mathrm{opt}}=\boldsymbol{G}
\end{array}\right\} .
$$

If noise is assumed to the planar wave and control sound is assumed to the spheric wave, the difference of both Distance-Attenuation and Time-Delay between the evaluation point and the original point of each filter are calculated from Eq. 3 as follows:

$$
\left.\begin{array}{l}
a_{d C(x y)}=\frac{l}{l_{s}} \\
a_{d D(x y)}=1 \\
a_{d K(x y)}=\frac{l_{s}}{l}
\end{array}\right\},
$$

From Eq. 5, the Time-Delay filters are calculated as

$$
\left.\begin{array}{l}
\boldsymbol{h}_{C(x y)}=\operatorname{IFFT}\left[\exp \left(-j \omega \Delta t_{C(x y)}\right)\right] \\
\boldsymbol{h}_{D(x y)}=\operatorname{IFFT}\left[\exp \left(-j \omega \Delta t_{D(x y)}\right)\right] \\
\boldsymbol{h}_{K(x y)}=\operatorname{IFFT}\left[\exp \left(-j \omega \Delta t_{K(x y)}\right)\right]
\end{array}\right\} .
$$

Equations 2, 4 and 6 are used for updating the filters.

The simulation was carried out in order to confirm the validity of the proposed method. The result is shown in Fig. 12. The control effect by optimal phase and by proposed method are almost the same. Optimal phase can be estimated without the prior identification if the position of evaluation point can be measured at each sampling time. More exactly adjusting gain characteristics is necessary in order to obtain better control effect.

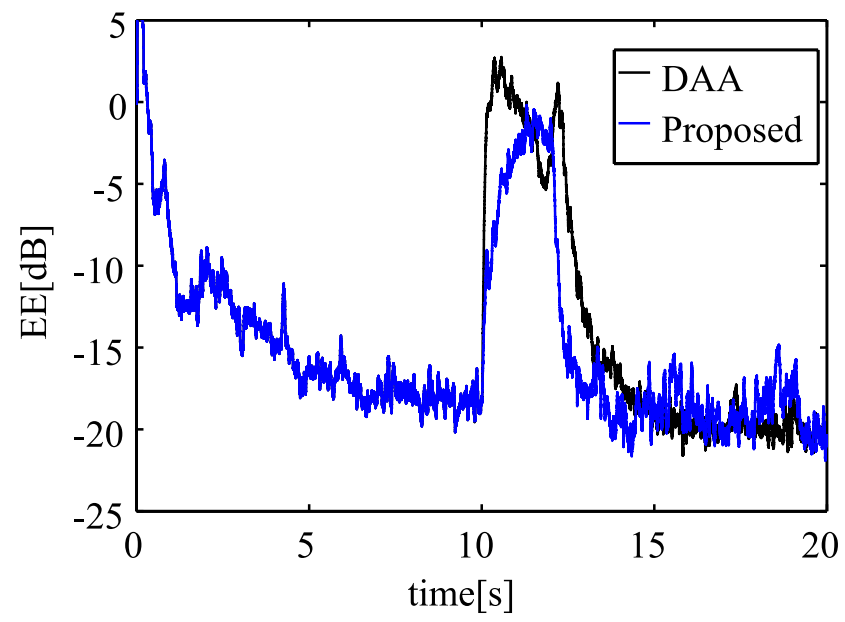

Fig. 12 A simulation controlled by the proposed method 


\section{Conclusion}

In this paper, the new algorithm for head-around ANC is proposed when the evaluation point moves. The measurements in $0.4 \mathrm{~m} \times 0.4 \mathrm{~m}$ area are carried out in order to investigate changes of acoustic characteristics when the evaluation point moves. Differences of acoustic characteristics are appeared in the difference of Time-Delay and that of amplitude. The identifications of optimal characteristics of controller are carried by computer simulations. The same as acoustic characteristics, differences of identified characteristics are appeared in Time-Delay and amplitude.

The simulation is carried out with interpolating the optimal filters. The enough control effect obtained. However, the prior identification is necessary to use this method. The simulation is carried out with the filters having the optimal phase characteristics. Compared with DAA, the better control effect obtained.

The new algorithm without previously identifying is proposed. This algorithm updates the filters as functions of location by calculating Time-Delay and Distance-Attenuation geometrically. The simulation result showed that the proposed algorithm and optimal phase characteristics control have almost the same effect. The gain characteristics should be adjusted exactly in order to obtain enough control effect.

\section{References}

( 1 ) Simon Haykin, “Adaptive Filter Theory”, Prentice-Hall, Inc.A Simon \& Scuster Company, 1996

( 2 ) Hiroshi IIDA, Mitsuru NAKAZAWA and Sekiya KOIKE, "Active Noise Control of a Moving Evaluation Point in One Dimensional Acoustic Field (3rd Report, Proposal of Time-delay Separation Method in the Secondary Path Identification)", Transactions of the Japan Society of Mechanical Engineers Series C, Vol.72 No.714 pp.102-108, 2006-2

( 3 ) Toshikazu KHONO, Yuhsuke OHTA and Akira SANO, "Adaptive Active Noise Control Algorithm without Explicit Identification of Secondary Path Dynamics", THE TRANSACTIONS OF THE INSTITUTE OF ELECTRONICS, INFORMATION AND COMMUNICATION ENGINEERS A, Vol.J86-A No.1 pp.9-18, 2003-1 